\title{
BIFURCATION PROBLEMS FOR NONCOMPACT OPERATORS
}

\author{
H. SALAHIFARD AND S. MANSOUR VAEZPOUR
}

Received 23 June, 2014

\begin{abstract}
Under new hypotheses, and using the Lyapunov-Schmidt reduction, we study the branches of bifurcation of a nonlinear equation of the type $u-\lambda L u+G(\lambda, u)=0$, in a neighborhood $W$ of a particular solution $\left(\lambda_{0}, 0\right) \in \mathbb{R} \times X$, where $X$ is a real Banach space, $L$ a noncompact linear operator defines on $X$ and $G$ is a nonlinear operator defined on $W$ to values in $X$. This type of bifurcation problems ( bifurcation from the trivial branch ) have different applications such as resolution of differential equations as those of Von-Karmann and Navier-Stokes or to integral equations as the Urysohn's one.
\end{abstract}

2010 Mathematics Subject Classification: 47H08; 47H11

Keywords: bifurcation problem, condensing map, Fredholm operators, $H$-set contractions

\section{INTRODUCTION}

Bifurcation from a trivial branch is one of the oldest notions of bifurcation in mathematics. In fact, assuming that there is a known (trivial) branch of solutions of a parameterized family of problems, necessary and sufficient conditions for the appearance of nontrivial solutions arbitrary close to some points (called bifurcation points) of the trivial branch is investigated. The most important examples of problems of bifurcation from the trivial branch come from nonlinear partial differential equations. For instance, see the von- Karman equations for the study of the buckling of thin plates [15]. More generally, those aspects of bifurcation theory which are particularly meaningful to differential equations are discussed in [5].

In what follows, we illustrate the procedure of bifurcation phenomenon from the simplest case. Assume that $X$ is a real Banach space and that $L$ is a compact linear operator on $X$. Set

$$
F(\lambda, x)=x-\lambda L x,
$$

then consider the problem of finding the pairs $(\lambda, x) \in \mathbb{R} \times X$ such that $F(\lambda, x)=0$. Let us take a look at the local structure of the zero set of $F$ around a given point $\left(\lambda_{0}, 0\right), \lambda_{0} \in \mathbb{R}$. If $\lambda_{0}$ is not a characteristic value of $L$, it is made up of exactly one curve (the trivial branch itself). If $\lambda_{0}=\lambda_{j}$ for some $j \geq 1$, the structure changes, since there are solutions of the form $\left(\lambda_{j}, x\right), x \in E_{j},\left(E_{j}\right.$ denotes the eigenspace associated with the characteristic value $\left.\lambda_{j}\right)$ arbitrarily close to $\left(\lambda_{j}, 0\right)$. The existence 
of nontrivial solutions (i.e. which do not belong to the trivial branch) around a point $\left(\lambda_{j}, 0\right)$ is referred to as a bifurcation phenomenon form the trivial branch and the points $\left(\lambda_{j}, 0\right)$ are called bifurction points.

Now consider the nonlinear equation

$$
x-\lambda L x+G(\lambda, x)=0,
$$

where $G: \mathbb{R} \times X \rightarrow X$ is an continuous operator. We recall that $\left(\lambda_{0}, 0\right)$ is a bifurcating point of (1.2) if every neighborhood of $\left(\lambda_{0}, 0\right)$ contains a nontrivial solution. Then the problem of bifurcation from the trivial branch of (1.2) turns into finding the zero set of the mapping

$$
F(\lambda, x)=x-\lambda L x+G(\lambda, x)=0,
$$

where $F: \mathbb{R} \times X \rightarrow X$.

Two reasons motivate this definition. First, problems of this type are common in many physical examples. Secondly, from a mathematical stand-point, their properties allow us to make a general study of their zero set. For the global analysis of (1.3), topological ideas play an essential role. When each $F(\lambda,$.$) lies in a class of operators$ for which an index is defined (for example, when $F(\lambda,$.$) is a compact perturbation of$ the identity, the Leray-Schauder degree and consequently the index is defined ), then a change in the degree of $F(\lambda,$.$) on a neighborhood of x=0$ as $\lambda$ passes through $\lambda_{0}$ implies that a bifurcation branch emanates from $\left(\lambda_{0}, 0\right)$, by using the index jump principle ([19] p.657). If there were no branching, by the homotopy property the degree would not change.

The most famous result about problems of bifurcation from the trivial branch is due to Krasnoselskii.

Theorem 1 ([7], Theorem 28.1). If $X$ is a real Banach space, $L$ is an compact operator on $X, \Omega \subseteq \mathbb{R} \times X$ a neighborhood of $\left(\lambda_{0}, 0\right), G: \Omega \rightarrow X$ is continuous and $G(\lambda, 0)=0$ and also

(i) $I-\lambda_{0} L$ is a Fredholm of index zero and $\lambda_{0}$ is an isolated characteristic value of $L$ of odd algebraic multiplicity,

(ii) $|G(\lambda, x)-G(\lambda, x)| \leq \varphi(r)|x-x|$ if $(\lambda, x),(\lambda, x) \in \Omega,\left|\lambda-\lambda_{0}\right| \leq \delta$ and $x, x \in$ $B_{r}(0)$ for some $\delta>0$ and some $\varphi: \mathbb{R}^{+} \rightarrow \mathbb{R}^{+}$such that $\varphi(r) \rightarrow 0$ as $r \rightarrow 0$.

Then $\left(\lambda_{0}, 0\right)$ is a bifurcation point for $F(\lambda, x)=x-\lambda L x+G(\lambda, x)=0$.

The proof of this theorem is based on topological degree arguments. It is a very general result but it does not provide any information on the structure of the zero set of $F(\lambda, x)$ near $\left(\lambda_{0}, 0\right)$.

Another important tool for the analysis of bifurcation from a trivial branch is the Lyapunov-Schmidt reduction, which leads a given bifurcation problem for integral and differential equations to a locally equivalent problem for a finite number of nonlinear equations in a finite number of indeterminates. It is worth mentioning that the choice of the approach depends on the nature of the problem at hand. 
One of the typical assumptions of the Lyapunov-Schmidt method is that singular points are isolated. Assuming this, there is a large variety of methods which, combined with the Lyapunov-Schmidt reduction, provide criteria for the appearance of nontrivial solutions close to the singular point $[4,6,11,12,17]$.

In 2002, Elhajji and Errachid [9], using the Lyapunov-Schmidt reduction, proved that the nonlinear equation (1.3) in a neighborhood $W$ of a particular solution $\left(\lambda_{0}, 0\right) \in$ $\mathbb{R} \times X$, admits parametric continuous curves formed by nontrivial solutions bifurcating from $\left(\lambda_{0}, 0\right)$ under the following assumptions:

(a) $X$ is a real Banach space,

(b) $L$ is a linear continuous and compact operator,

(c) $\lambda_{0} \in \mathbb{R}$ is a simple characteristic value of $L$, such that

$$
X=N\left(I-\lambda_{0} L\right) \oplus R\left(I-\lambda_{0} L\right),
$$

where $R($.$) and N($.$) denoted the range and null space of an operator respectively and$ $I$ is the identity map.

(d) $G: W \rightarrow X$ is a nonlinear operator of perturbation such that $G$ is $\alpha$-regular ( for $\alpha \in(0,1])$ in a neighborhood of $\left(\lambda_{0}, 0\right)$, (see the definition of $\alpha$-regular in Section 4).

In fact they provided a general convergent iterative procedure to compute the desired solutions $(\lambda, u)$ near $\left(\lambda_{0}, 0\right)$. The case $\alpha=1$ has been studied earlier by Berger and Westreich [3].

In 2005, Kim [13] generalized the Krasnoselskii's Theorem by defining a coincidence degree for a pair $(A, L)$ of operators, where $L: \mathbb{W} \rightarrow \mathbb{W}$ is compact and $A: \mathbb{W} \rightarrow \mathbb{W}$ is a condensing perturbation of the identity (see the definition of condensing operators below). She investigated the solvability of the bifurcation problem $A u+\lambda L u+G(\lambda, u)=0$, where $G: \mathbb{R} \times \mathbb{W} \rightarrow \mathbb{W}$ is a nonlinear perturbation such that $\|G(\lambda, u)\| \leq k\|u\|$ for every $(\lambda, u) \in \mathbb{R} \times \mathbb{W}$ and for some $k>0$. So in case $A$ is identity one can get the Krasnoselskii's Theorem in Hilbert spaces.

To the best of our knowledge, there is no paper studying such bifurcation problems when $L$ is not a compact operator. In this paper, we will consider the same bifurcation problem of (1.3) in a more general setting under some appropriate assumptions. It is worth mentioning that assumption (1.4) above is always satisfied if $X$ is a Hilbert space and if $L$ has closed range and is self-adjoint. For a real Banach space $X$, Assumption (1.4) holds if $\lambda_{0}$ is a simple characteristic value of linear compact operator $L$ and $I-\lambda_{0} L$ is a Fredholm operator of index zero. Here we provide some conditions for a noncompact operator $L$ on a Banach space $X$ such that (1.4) is satisfied.

We begin by recalling some definitions and preliminaries.

Let $(X, d)$ be a metric space and $B$ is a bounded subset of $X$, Kuratowski has defined $\alpha(B)$, the measure of noncompactness of $B$, to be

$\alpha(B)=\inf \{r>0: B$ may be covered by finitely many sets of diameter $\leq r\}$. 
It is convenient in applications to generalize slightly the idea of measure of noncompactness.

A function $\gamma:\{B \subset X: B$ is bounded $\} \rightarrow[0, \infty)$ is said to be a generalized measure of noncompactness on a Banach space $X$, if it satisfies the following conditions:

(1) (invariance under closure and convex hull): $\gamma(\overline{c o B})=\gamma(B)$,

(2) (regularity): $\gamma(B)=0$ if and only if $B$ is relatively compact,

(3) (semi-additivity): $\gamma\left(B_{1} \cup B_{2}\right)=\max \left\{\gamma\left(B_{1}\right), \gamma\left(B_{2}\right)\right\}$,

(4) (algebraic semi-additivity): $\gamma\left(B_{1}+B_{2}\right) \leq \gamma\left(B_{1}\right)+\gamma\left(B_{2}\right)$

(5) (semi-homogeneity): $\gamma(\alpha B)=|\alpha| \gamma(B)$ for all $\alpha \in R$.

The definition of generalized measure of noncompactness given above is more general than Kuratowski or the other definition of measure of noncompactness, namely Hausdorff measure of noncompactness (or ball measure of noncompactness $[1,2,18]$ ) given by

$$
\beta(B)=\inf \{r>0 \text { : there exists a finite } r \text {-net for } B \text { in } X\} \text {. }
$$

A continuous operator $T: X \rightarrow X$ is said to be:

- countably $k$-set contraction (with respect to generalized measure of noncompactness $\gamma$ ) [14]:

if $\gamma(T(C)) \leq k \gamma(C)$ for each countable bounded set $C \subseteq X$ and $0 \leq k<1$,

- countably condensing if $\gamma(T(C))<\gamma(C)$ for each countable bounded set $C \subset X$ with $\gamma(C)>0$.

- countably $\varphi$-set contraction [8]:

if $\gamma(T(C)) \leq \varphi(\gamma(C))$ for some $\varphi \in \Phi=\left\{\varphi: R^{+} \rightarrow R^{+}, \varphi(t)<t\right.$ for $t>0, \varphi(0)=$ $0\}$ and each countable bounded set $C \subseteq X$.

Clearly, every $k$-set contractive mapping is a $\varphi$-set contractive where $\varphi(t)=k t$. Let $T$ be a bounded linear operator of a Banach space $X$ into a Banach space $Y$. The null space and the range of $T$ are denoted by $N(T)$ and $R(T)$, respectively. $T$ is said to be a Fredholm operator if the dimension of $N(T)$ is finite and the codimension of $R(T)$ is finite. If the dimension of $N(T)$ is finite and $R(T)$ is closed, then $T$ is said to be a semi-Fredholm operator. In this case, $i(T)=\operatorname{dim} N(T)-\operatorname{codim} R(T)$ is called the index of $T$.

\section{H-SET CONTRACTIONS}

In this section we state our main definition which determines an important class of operators.

Definition 1. Let $H: \mathbb{R}^{+} \rightarrow \mathbb{R}$ be a mapping satisfying:

(H1) $H$ is strictly increasing, i.e. $H(\alpha)<H(\beta)$ for $\alpha<\beta \quad\left(\alpha, \beta \in \mathbb{R}^{+}\right)$.

(H2) $H(\alpha . \beta) \leq H(\alpha)+H(\beta)$.

A mapping $T: X \rightarrow X$ is said to be countably $H$-set contraction if there exists $\tau>0$ 
such that for all countable bounded set $C \subseteq X$

$$
(\gamma(T(C))>0) \Rightarrow \tau+H(\gamma(T(C))) \leq H(\gamma(C)) .
$$

Remark 1. From (H1) and (2.1) it is easy to conclude that every $H$-set contraction is a condensing map.

Example 1. Let $H: \mathbb{R}^{+} \rightarrow \mathbb{R}$ be given by $H(x)=\ln x$. It is clear that $H$ satisfies $(H 1)$ and $(H 2)$. Each Mapping $T: X \rightarrow X$ satisfying (2.1) is an $H-$ set contraction such that

$$
\gamma(T(C)) \leq e^{-\tau} \gamma(C)
$$

for all countable set $C \subset X$ with $\gamma(T(C))>0$. It is clear that for a countable set $C \subset X$ if $\gamma(T(C))=0$ the inequality (2.2) is also holds, i.e. $T$ is a countably $k-$ set contraction with $k=e^{-\tau}$.

Example 2. Let $H: \mathbb{R}^{+} \rightarrow \mathbb{R}$ be given by $H(x)=\ln \left(x^{2}+x\right)$. Obviously $H$ satisfies (H1) and (H2) and for $H$-set contraction $T$ and countable set $C \subset X$ with $\gamma(T(C))>0$, the following condition holds:

$$
\frac{\gamma(T(C))(\gamma(T(C))+1)}{\gamma(C)(\gamma(C)+1)} \leq e^{-\tau}
$$

Example 3. Let $H:[1, \infty) \rightarrow \mathbb{R}$ be given by $H(x)=\frac{-1}{\sqrt{x}}+1$. Then $H$ satisfies (H1) and (H2) and for $H$-set contraction $T$, we have

$$
\gamma(T(C)) \leq \frac{1}{(1+\tau \sqrt{\gamma(C)})^{2}} \gamma(C),
$$

for all countable set $C \subset X$ with $\gamma(T(C))>0$. Here we obtained a special case of $\varphi-$ set contractions.

\section{MAIN RESULTS}

Lemma 1. Let $X$ be a real or complex Banach space and $T: X \rightarrow X$ a bounded linear operator and $T^{n}$ is countably condensing for some $n \in N$. Let I denote the identity operator in $X$. Then $I-T$ is a semi-Fredholm operator.

Proof. First, we will show that the range of $I-T$ is closed in $X$. Let $M$ and $C$ be relatively compact set and bounded countable set in $X$ respectively. Set $M_{1}=$ $\{x \in C: x-T x \in M\}$. We will show that $\gamma\left(M_{1}\right)=0$. Suppose that $x \in M_{1}$, so that $x=T x+z$ for some $z \in M$. Substituting for $x$ on the right, $x=T^{2} x+T z+z$, and continuing in this way we find

$$
x=T^{n} x+\left(\Sigma_{j=0}^{n-1} T^{j}\right) z .
$$

If we write $M_{2}=\left(\sum_{j=0}^{n-1} T^{j}\right)(M)$, the set $M_{2}$ is relatively compact because it is the continuous image of a relatively compact set. Furthermore, the above equality 
implies that $M_{1} \subset T^{n}\left(M_{1}\right)+M_{2}$, so that $\gamma\left(M_{1}\right) \leq \gamma\left(T^{n} M_{1}\right)$. Since $T^{n}$ is condensing, from $\gamma\left(M_{1}\right)>0$ we get a contradiction, therefore $\gamma\left(M_{1}\right)=0$ and thus $M_{1}$ is relatively compact.

Now it suffices to show that $I-T$ maps bounded closed sets into closed sets. Let $D$ be any bounded closed set in $X$. Suppose that $\left\{x_{n}\right\}$ is a sequence in $D$ such that $y_{n}=x_{n}-T x_{n} \rightarrow y$ for some $y \in X$. Since $\left\{y_{n}\right\}$ is relatively compact, by the above argument $\left\{x_{n}\right\}$ is relatively compact. Therefore there exist a subsequence $\left\{x_{n_{k}}\right\}$ and an $x \in D$ such that $x_{n_{k}} \rightarrow x$ as $k \rightarrow \infty$. By the continuity of $T$, we obtain that $x_{n_{k}}-T x_{n_{k}} \rightarrow x-T x$ as $k \rightarrow \infty$ and hence $y=x-T x \in(I-T) D$. We conclude that $(I-T) D$ is closed in $X$.

Now we show that the null space of $I-T$ is finite dimensional. Assume that $N(I-T)$ is infinite dimensional. Let $S=\{x \in N(I-T):\|x\|=1\}$. It is clear that $S$ would not be compact in the infinite dimensional Banach space $N(I-T)$, hence there would exist a sequence $\left\{x_{k}\right\}$ in $S$ such that $\left\|x_{k}-x_{l}\right\|>\frac{1}{2}$ for all $k \neq l$. Since $T^{n}$ is countably condensing and $\gamma\left(\left\{x_{k}\right\}\right)>0$, we would have $\gamma\left(\left\{x_{k}\right\}\right)=\gamma\left(\left\{T^{n} x_{k}\right\}\right)<$ $\gamma\left(\left\{x_{k}\right\}\right)$, which is a contradiction. We conclude that $I-T$ is a semi- Fredholm operator.

Let $X$ be a real or complex Banach space and $T: X \rightarrow X$ a bounded linear operator. Set

$$
\begin{aligned}
\tau_{n} & =\sup \left\{\tau>0: T^{n} \text { is an } H-\text { set contraction }\right\}, \\
\gamma_{H}\left(T^{n}\right) & =H^{-1}\left(-\tau_{n}\right), \\
r_{H}(T) & =i n f_{n \geq 1}\left(\gamma_{H}\left(T^{n}\right)\right)^{\frac{1}{n}} .
\end{aligned}
$$

Theorem 2. If $\mu \in \mathbb{C}$ be such that

$$
H\left(|\mu|^{n}\right)<\tau_{n} \text { for some } n \in \mathbb{N},
$$

then $I-\mu T$ is a Fredholm operator of index zero.

Proof. Consider an operator $T_{1}=\mu T: X \rightarrow X$ and notice that

$$
\begin{aligned}
H\left(\gamma\left(T_{1}^{n}(C)\right)\right) & =H\left(\gamma(\mu T)^{n}(C)\right) \\
& \leq H\left(|\mu|^{n}\right)+H\left(\gamma\left(T^{n}(C)\right)\right) .
\end{aligned}
$$

Since $H\left(|\mu|^{n}\right)<\tau_{n}$ for some $n \in \mathbb{N}$ one can conclude that

$$
H\left(\gamma\left(T_{1}^{n}(C)\right)\right)<H(\gamma(C)),
$$

for some $n \in \mathbb{N}$. Since $H$ is strictly increasing we have

$$
\gamma\left(T_{1}^{n}(C)\right)<\gamma(C)
$$


i.e. $T_{1}^{n}$ is countably condensing for some $n \in \mathbb{N}$, so by previous Lemma $I-T_{1}$ is semi- Fredholm operator. Note that for any $t \in[0,1]$,

$$
\gamma\left(\left(t T_{1}\right)^{n}(C)\right)=t^{n} \gamma\left(T_{1}^{n}(C)\right) \leq \gamma\left(T_{1}^{n}(C)\right)<\gamma(C) .
$$

i.e. $\left(t T_{1}\right)^{n}$ is countably condensing for some $n \in \mathbb{N}$. Indeed by the same argument $I-t T_{1}$ is a semi-Fredholm operator for each $t \in[0,1]$. Since $\left\{I-t T_{1}: t \in[0,1]\right\}$ is connected and the index for semi-Fredholm operators is constant on its connected components, we have

$$
i(I-\mu T)=i\left(I-T_{1}\right)=i(I)=0 .
$$

Thus, $I-\mu T$ is a Fredholm operator of index zero.

Lemma 2. If $\mu \in \mathbb{C}$ be such that

$$
\left(H^{-1}\left(-H\left(|\mu|^{n}\right)\right)^{\frac{1}{n}}>r_{H}(T) \text { for } n \in \mathbb{N}\right.
$$

then (3.1) will be satisfied.

Proof. By definition of $r_{H}(T)$ one can choose an integer $n_{0} \in \mathbb{N}$ such that

$$
\left(H^{-1}\left(-H\left(|\mu|^{n}\right)\right)^{\frac{1}{n}}>\left(\gamma_{H}\left(T^{n_{0}}\right)\right)^{\frac{1}{n_{0}}},\right.
$$

so

$$
H^{-1}\left(-H\left(|\mu|^{n_{0}}\right)\right)>\left(\gamma_{H}\left(T^{n_{0}}\right)\right)=H^{-1}\left(-\tau_{n_{0}}\right) .
$$

From property (H1) of (1), $H$ and also $H^{-1}$ are strictly increasing, so we have

$$
-H\left(|\mu|^{n_{0}}\right)>-\tau_{n_{0}},
$$

therefore

$$
H\left(|\mu|^{n_{0}}\right)<\tau_{n_{0}}
$$

for some $n_{0} \in \mathbb{N}$.

Remark 2. Let $H(x)=\ln x, \gamma_{c}\left(T^{n}\right)=\inf \left\{k \geq 0: T^{n}\right.$ is an $k$-set contraction $\}$ and $r_{c}(T)=\inf _{n \geq 1}\left(\gamma_{c}\left(T^{n}\right)\right)^{\frac{1}{n}}$ (see [14]), then the left hand side of condition (3.2) is equal to $\frac{1}{|\mu|}$ and the right hand side of it is $r_{c}(T)$, i.e. (3.2) is equivalent to $|\mu| r_{c}(T)<$ 1. So by Lemma 2 if $\mu \in \mathbb{C}$ such that $|\mu| r_{c}(T)<1$, then (3.1) will be satisfied. Moreover if $\mu \in \mathbb{C}$ such that $|\mu| \gamma_{c}(T)<1$, then since $\gamma_{H}(T) \geq r_{H}(T)$, again (3.1) will be satisfied.

Corollary 1. Let $T: X \rightarrow X$ be a bounded linear operator. If $\mu \in \mathbb{C}$ such that

$$
\left(H^{-1}\left(-H\left(|\mu|^{n}\right)\right)\right)^{\frac{1}{n}}>\gamma_{H}(T) \text { for } n \in \mathbb{N}
$$

then $I-\mu T$ is a Fredholm operator of index zero. 


\section{SOlVABILITY OF THE BIFURCATION PROBLEM}

In 2002, Elhajji and Errachid [9], proved that the nonlinear equation

$$
u-\lambda L u+G(\lambda, u)=0,
$$

in a neighborhood $W$ of a particular solution $\left(\lambda_{0}, 0\right) \in \mathbb{R} \times X$, admits parametric continuous curves formed by nontrivial solutions bifurcating from $\left(\lambda_{0}, 0\right)$ under the main assumption:

$$
X=N\left(I-\lambda_{0} L\right) \oplus R\left(I-\lambda_{0} L\right) .
$$

This decomposition for a compact operator $L$ implies the existence of linear canonical bounded projections $p$ and $q$ of $X$ onto $N\left(I-\lambda_{0} L\right)$ and $R\left(I-\lambda_{0} L\right)$, respectively. In fact they provided a general convergent iterative procedure to compute the nontrivial solutions $\left(\lambda, u=\varepsilon u_{0}+v\right)$ near $\left(\lambda_{0}, 0\right)$ by projecting (4.1) onto $N\left(I-\lambda_{0} L\right)$ and $R\left(I-\lambda_{0} L\right)$.

Now, motivated by them we provide some condition in order to decompose the Banach space $X$ as (4.2) and then we solve the bifurcating problem (4.1) for noncompact operator $L$.

Theorem 3. Let

(1) $X$ be a real Banach space,

(2) The linear continuous operator $L: X \rightarrow X$ be an $H$-set contraction,

(3) $\lambda_{0} \in \mathbb{R}$ be such that $H\left(\left|\lambda_{0}\right|^{n}\right)<\tau_{n}$ for some $n \in \mathbb{N}$, and $N\left(I-\lambda_{0} L\right) \cap R(I-$ $\left.\lambda_{0} L\right)=\{0\}$.

(4) A nonlinear operator $G: W \rightarrow X$ is $\alpha$-regular ( $\alpha$ being a real parameter in $(0,1])$ in a neighborhood of $\left(\lambda_{0}, 0\right)$ i.e. there is $M>0$ such that

$$
\begin{aligned}
& \|G(\lambda, u)-G(\lambda, \dot{u})\| \leq M\left(\|u\|^{\alpha}+\|\|^{\alpha}\right)\|u-\dot{u}\|, \quad(\lambda, u),(\lambda, u) \in W . \\
& \|G(\lambda, u)-G(\dot{\lambda}, u)\| \leq M\|u\|^{\alpha+1}|\lambda-\hat{\lambda}|, \quad(\lambda, u),(\dot{\lambda}, u) \in W \text {. }
\end{aligned}
$$

then the problem (4.1) admits a unique parametric continuous curve $\varepsilon \in\left[-\varepsilon_{0}, \varepsilon\right] \mapsto$ $\left[\lambda(\varepsilon), u(\varepsilon)=\varepsilon u_{0}+v(\varepsilon)\right]$ formed by nontrivial solutions bifurcating with the trivial one $(\lambda, 0)$ from $\left(\lambda_{0}, 0\right)$, with $\lambda(0)=0, v(0)=0$ and verifying

$$
\begin{aligned}
\left|\lambda(\varepsilon)-\lambda_{0}\right| & =O\left(|\varepsilon|^{\alpha}\right) \\
|| v(\varepsilon)|| & =O\left(|\varepsilon|^{\alpha+1}\right)
\end{aligned}
$$

Proof. Let $\lambda_{0} \in \mathbb{R}$ be such that $H\left(\left|\lambda_{0}\right|^{n}\right)<\tau_{n}$ for some $n \in \mathbb{N}$, then by Theorem 2, $I-\lambda_{0} L$ is a Fredholm operator of index zero. Since $N\left(I-\lambda_{0} L\right) \cap R\left(I-\lambda_{0} L\right)=$ $\{0\}$, one can write $X=N\left(I-\lambda_{0} L\right) \oplus R\left(I-\lambda_{0} L\right)$. Now the desired result follows immediately from Theorem 3 in [9]. 


\section{APPLICATION}

As an application of Theorem 3 for which the case for compact maps can not be applied we consider the Hartree equation for the Helium atom (See [16]). The Hartree equation is of the form

$$
u-\lambda\left[u \int \frac{u^{2}(t)}{|t-x|} d t+\frac{2}{\|x\|} u+\frac{1}{2} \Delta u\right]=0
$$

and can be represented in form $u-\lambda T(u)=0$ where $T$ is not compact. Since $T$ is of the form $B+C$ where $B$ is compact and $C$ is an $H$-set contraction with $H(x)=\ln x$ and $\tau=\ln \frac{1}{M}$ where $M \rightarrow 0^{+}$in a neighborhood of the origin in which $T$ is an $H$-set contraction.

Another example of $H$-set contractions which often appears in the study of neutral systems is the following model for an Hopfield neural networks system: set

$$
Y=C_{\omega}=\left\{x(t)=\left(x_{1}(t), \ldots, x_{n}(t)\right) \in C\left(\mathbb{R}, \mathbb{R}^{n}\right): x_{i}(t+\omega) \equiv x_{i}(t), i=1, \ldots, n\right\},
$$

with the norm defined by $|x|_{0}=\sum_{i=1}^{n} \max _{t \in[0, \omega]}\left|x_{i}(t)\right|$, and $X=C_{\omega}^{1}=\left\{x(t)=\left(x_{1}(t), \ldots, x_{n}(t)\right) \in C^{1}\left(\mathbb{R}, \mathbb{R}^{n}\right): x_{i}(t+\omega) \equiv x_{i}(t), i=1, \ldots, n\right\}$, with the norm $\|x\|=\max \left\{|x|_{0},|\dot{x}|_{0}\right\}$. Then $C_{\omega}, C_{\omega}^{1}$ are Banach spaces. Define $N: C_{\omega}^{1} \rightarrow C_{\omega}$ by

$$
N\left(x_{i}\right)=-b_{i} x_{i}+\sum_{j=1}^{n} a_{i j} g_{j}\left(x_{j}\left(t-s_{i j}(t)\right)\right)+\sum_{j=1}^{n} b_{i j} g_{j}\left(\dot{x}_{j}\left(t-r_{i j}(t)\right)\right)
$$

where $i=1,2, \ldots, n, x_{i}(t)$ corresponds to the state of the $i$ th unit at time $t, b_{i}>0$ denotes the neuron firing rate, $a_{i j}$ and $b_{i j}$ represent the delayed connection weight and neutral delayed connection weight, respectively. $s_{i j}(t), r_{i j}(t) \geq 0$ is the transmission delay, and $g_{j}$ is the activation function in the system. By assuming some conditions on $g, s, r\left(\operatorname{see}\left(H_{1}\right)-\left(H_{3}\right)[10]\right)$ one can show that if $M=\max \left\{\sum_{j=1}^{n}\left|b_{i j}\right| L_{j}, i=\right.$ $1, \ldots, n\}<1$ then $N$ is an $H$-set contraction with $\tau=-\ln M$. Since the proof is similar to Lemma 3 in [10] it will be omitted here.

\section{REFERENCES}

[1] R. R. Akhmerov, M. I. Kamenskii, A. S. Patapov, A. E. Rodkina, and B. N. Sadovskii, Measures of noncompactness and condensing operators, Operator Theory, 2nd ed., ser. Advances and Applications. Basel: Birkhäuser Verlag, 1992, vol. 55.

[2] J. Appel, "Measures of noncompactness, condensing operators and fixed points: an appicationoriented survey," Fixed Point Theory, vol. 6, no. 2, pp. 157-229, 2005.

[3] M. Berger and D. Westreich, "A convergent iteration scheme for bifurcation theory in Banach spaces," J. Math. Anal. Appl., vol. 43, pp. 136-144, 1973, doi: 10.1016/0022-247X(73)90263-1.

[4] B. Buffoni and J. Toland, Analytic theory of global bifurcation. An introduction, ser. Princeton Series in Applied Mathematics. Princeton: Princeton University Press, 2003.

[5] S. N. Chow and J. K. Hale, Methods of Bifurcation Theory, ser. Texts in Applied Mathematics. New York: Springer-Verlag, 1982, vol. 34, doi: 10.1007/978-1-4613-8159-4. 
[6] B. Darinskii, Y. Sapronov, and S. Tsarev, "Bifurcation of extremals of Fredholm functionals," Journal of Mathematical Sciences, vol. 145, pp. 5311-5445, 2007, doi: 10.1007/s10958-0070356-2.

[7] K. Deimling, Nonlinear Functional Analysis. Berlin: Springer, 1985. doi: 10.1007/978-3-66200547-7.

[8] B. C. Dhage, "Condensing mappings and applications to existence theorems for common solution of differential equations," Bull. Korean Math. Soc., vol. 36, no. 3, pp. 565-578, 1999.

[9] S. Elhajji and M. Errachid, "Analysis of a bifurcation problem," Mathematics and Computers in Simulation, vol. 58, pp. 231-245, 2002, doi: 10.1016/S0378-4754(01)00366-4.

[10] Z. Gui, W. Gec, and X.-S. Yang, "Periodic oscillation for a Hopfield neural networks with neutral delays," Physics Letters A, vol. 364, pp. 267-273, 2007, doi: 10.1016/j.physleta.2006.12.013.

[11] J. Ize and A. Vignoli, Equivariant degree theory. Berlin: Walter de Gruyter and Co., 2003. doi: $10.1515 / 9783110200027$.

[12] H. Kielhöfer, Bifurcation theory. An introduction with applications to PDEs, ser. Applied Mathematical Sciences. New York: Springer-Verlag, 2004, vol. 156.

[13] I. S. Kim, “A solvability of bifurcation problems," Nonlinear Anal, vol. 63, pp. 1809-1816, 2005, doi: 10.1016/j.na.2004.12.025.

[14] I. S. Kim, "Index formulas for countably $k$-set contractive operators," Nonlinear Analysis, vol. 69, pp. 4182-4189, 2008, doi: 10.1016/j.na.2007.10.046.

[15] P. Rabier, Lectures on Topics In One-Parameter Bifurcation Problems. Berlin, Heidelberg, New York, Tokyo: Springer-Verlag, 1985.

[16] M. Reeken, "A general theorem on bifurcation and its application to the hartree equation of the helium atom," J. Math. Physics, vol. 11, pp. 2505-2512, 1970, doi: 10.1063/1.1665418.

[17] D. H. Sattinger, Group theoretic methods in bifurcation theory, Lecture Notes in Math. Berlin, Heidelberg: Springer, 1979, vol. 762.

[18] J. M. A. Toledano, T. D. Benavides, and G. L. Acedo, Measures of Noncompactness in Metric Fixed Point Theory. Basel: Birkhäuser, 1997.

[19] E. Zeidler, Nonlinear Functional Analysis and its Applications. Springer- Verlagl, 1986.

Authors' addresses

H. Salahifard

Amirkabir University of Technology, Department of Mathematics and Computer Sciences, Hafez

Av., Tehran, Iran

E-mail address: salahifardegmail.com

S. Mansour Vaezpour

Amirkabir University of Technology, Department of Mathematics and Computer Sciences, Hafez Av., Tehran, Iran

E-mail address: vaez@aut.ac.ir 